\title{
Kinematics Analysis on Cubic Mechanism with Auxiliary Joints
}

\author{
Jianguo Luo* \\ College of Mechanical \& Electrical Engineering, \\ North China Institute of Science and Technology \\ Beijing, 101601, China \\ *Corresponding author
}

\author{
Maoyan He \\ Labour Union \\ North China Institute of Science and Technology \\ Beijing, 101601, China
}

\author{
Yuanyuan Liu \\ College of Mechanical \& Electrical Engineering, \\ North China Institute of Science and Technology \\ Beijing, 101601, China
}

\begin{abstract}
A new type of hybrid cubic manipulator with six degree of freedom(DOF) suggested based on traditional serial manipulator and parallel manipulator, analytic geometry method adopted to find its forward solution of position, simple expression and exclusive result obtained, as well as its inverse solution of position discussed here, still exclusive solution found. Making use of software to simulate the manufacturing of a round trajectory, position and velocity and acceleration of each input parameter obtained, which can be verified through numerical computation, each input parameter decoupled very well, which greatly simplify the kinematics control of mechanism, perfect machine tool and so on can be developed based on the mechanism, research on the kinematics of this mechanism can provide reference to the practical application of it.
\end{abstract}

Keywords-hybrid manipulator; analytical geometry; forward solution; inverse solution; simulation

\section{INTRODUCTION}

There are two basic problems in the kinematics of mechanism, namely problem of forward solution and inverse solution of mechanism. The forward solution and inverse solution of position of mechanism is the basic task of kinematics analysis of mechanism, it is also the basis of analysis on velocity and acceleration and error and kinetics and synthesis of mechanism[1]. Zhang[2] research on a 4-UPS-CPC parallel mechanism, establish a mechanism model and a mathematical model, position and velocity and acceleration analyzed, mapping equation of input and output velocity and acceleration established then, based on the kinematics model, mathematical software used to verify the correct of the kinematical solution, still simulation adopted to verify the feasibility of experiment. Chen[3] and Song[4] and Yang[5] researched on 4-UPS-RPS parallel mechanism and a bionic robot and planar six bar mechanism with mathematical and simulation methods respectively. In order to expand the structure form and scope of application of serial-parallel manipulator, also to improve its performance, a new type of hybrid cubic robot proposed in this paper, six degree of freedom realized by three dimension spatial linear drive control. New type of hybrid cubic mechanism with six degree of freedom, the general form of the kinematic solution obtained through forward and inverse kinematics analysis, displacement and velocity and acceleration found through kinematical simulation with determined trajectory, by comparing the analytical results, spatial kinematics performance of mechanism can be expressed intuitively, still its superiority in kinematic analysis found, which lay the foundation for following research and development.

\section{INVERSE KINEMATICS ANALYSIS AND SIMULATION}

Method on forward solution mainly includes analytical method and numerical method[6]. The analytic method is mainly through the elimination of the unknowns in the constraint equations of mechanism, thus making the input and output equation of the mechanism to be a higher order equation containing only one unknown quantity. Numerical algorithm is a method that given an initial value, cycle started from the initial value, the iteration close to the given input value, until the degree meet the required accuracy[7]. Fixed coordinate system construct on PPP-S(P)[T]-PPP new type of hybrid mechanism, as shown in Fig.1. Spherical joint and Hooke joint connected by passive telescopic joint $\mathrm{P}$, which provide an auxiliary adaptive function for the length change of output shaft, new hybrid cubic mechanism can realize six degree of freedom movement with three-dimensional translational motion and three-dimensional rotation.

Assume the position and pose of output shaft determined, construct coordinate system $\mathrm{Oxyz}$ as shown in Figure 1, traditional method to get the inverse solution of position of parallel mechanism adopt to find the expression containing the Jacobin matrix $\mathbf{J} x=b$, so as to obtain the inverse solution. Analytic method used to find its inverse solution of position here. Suppose the position of center point of output moving platform and pose of output shaft, $\left(x_{P}, y_{P}, z_{P}\right)$ is 
the coordinates of output shaft end point $\mathrm{P},(u, v, w)$ is the direction vector of axis $n$, remaining parameters are the same to the definition in the process of forward solution, due to the special structure of mechanism, the coordinates of center point of hook joint and spherical joint can be written as following respectively,

$$
\begin{aligned}
& O_{1}:\left\{\begin{array}{l}
x_{O_{1}}=x_{P}-\frac{t}{h} \cdot u \\
y_{O_{1}}=y_{P}-\frac{t}{h} \cdot v \\
z_{O_{1}}=z_{P}-\frac{t}{h} \cdot w \\
y_{O_{2}}=y_{P}-\left(\frac{t}{h}-1\right) \cdot v \\
x_{O_{2}}=x_{P}-\left(\frac{t}{h}-1\right) \cdot u
\end{array}\right. \\
& z_{P}-\left(\frac{t}{h}-1\right) \cdot w
\end{aligned}
$$

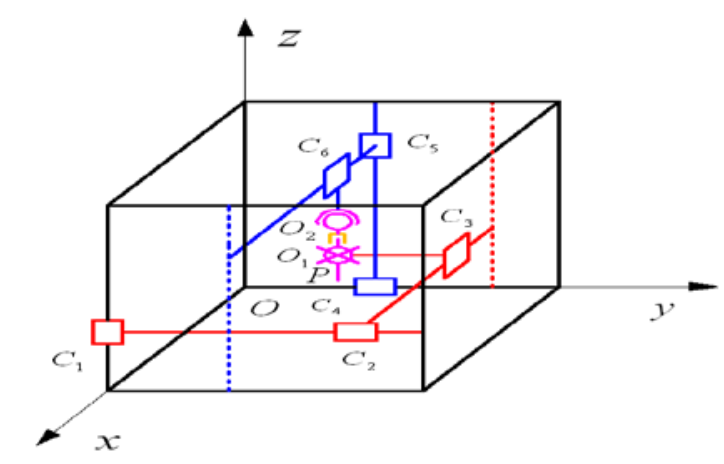

FIGURE I. PPP-S (P) [T]-PPP SPATIAL CUBIC MECHANISM

As we can see from the expression (1) to (2), the coordinates of each input point of each input slider can be obtained, there exist $C_{1}:\left(a, 0, z_{O_{1}}\right)$, $C_{2}:\left(a, y_{O_{1}}+d_{1}, z_{O_{1}}\right) \quad, \quad C_{3}:\left(x_{O_{1}}, y_{O_{1}}+d_{1}, z_{O_{1}}\right)$, $C_{4}:\left(0, y_{\mathrm{O}_{2}}, \mathrm{O}\right) \quad, \quad C_{5}:\left(0, y_{\mathrm{O}_{2}}, z_{\mathrm{O}_{2}}+d_{2}\right)$, $C_{6}:\left(x_{\mathrm{O}_{2}}, y_{\mathrm{O}_{2}}, z_{\mathrm{O}_{2}}+d_{2}\right)$, inverse solution of mechanism found then, if the position and velocity and acceleration of the output end of the mechanism is given, the position and velocity and acceleration of each input slider can be obtained.

\section{CALCULATION EXAMPLES}

According to the results of inverse kinematics analysis, with the intuitive and effective results from computer analysis and simulation, the correctness of calculation and analysis is verified. Relative parameters given as follows, $a=1000 \mathrm{~mm}, \quad t=100 \mathrm{~mm}, \quad h=200 \mathrm{~mm}$, $d_{1}=200 \mathrm{~mm}, d_{2}=100 \mathrm{~mm}$, it is assumed that the vertical height of point $P$ on output shaft is $300 \mathrm{~mm}$ in cubic mechanism, with point $S:(500,500,300)$ as the center of the circle, which move around the vertical coordinate axis $\mathrm{Oz}$ with radius $200 \mathrm{~mm}$, output shaft $\mathrm{O}_{1} \mathrm{O}_{2}$ always keep the relationship of cosine angle equal to $30^{\circ}$ with coordinate axis $\mathrm{Oz}$, circumferential speed of the initial position of point Pis $\omega_{P_{0}}=\frac{\pi}{18}(\mathrm{rad} / \mathrm{s})$, its angular acceleration is $\delta_{P}=0.1\left(\mathrm{rad} / \mathrm{s}^{2}\right)$, the circular motion step of output end point $P$ is defined as $5^{\circ}$, its moving range is defined as $\left[0,360^{\circ}\right]$, as shown in Figure 2. Kinematical properties of each input sliders verified by results of analysis and simulation as shown in Figure 3 to Figure 8.

\section{CONCLUSION}

New cubic hybrid mechanism include upper and lower symmetrical structure, six degree of freedom can be obtained, which can be realized by six linear drive, perfect decoupling characteristics as well, the forward kinematics solution determined uniquely, and the form is simple, provide the direct basis for the application of mechanism. The inverse kinematics solution determined uniquely as well, simulation of manufacturing a circle trajectory realized by making use of computer software for simulation, spatial displacement and velocity and acceleration curves of each input sliders obtained, the results completely consistent to the the results from mathematical calculating method, which can provide a good reference for its actual development and application based on the research of kinematic characteristics in theory.

\section{ACKNOWLEDGEMENT}

This research was financially supported by the HeBei Province Science and Technology Foundation (No.Z2015072) and NCIST Foundation(No. 3142015023).

\section{REFERENCES}

[1] B. Zhou, Y. Xu, "Robust control of hybrid robot,” Journal of Hunan University, 2005, Vol.12, pp.45-49.

[2] Y. M. Huang, F. Gao, and W. B. Shi, "Development in CNC series-parallel machine tool,” Manufacturing Technology \& Machine Tool, 2001, Vol.8, pp.8-9.

[3] W. Zhao, J. J. Yu, and S. S. Bi, "Experimental system of serial-parallel micromanipulator," Jouranl of Beijing University of Aeronautics and Astronautics, 2001, Vol. 6, pp.47-49.

[4] L. J. Xu, S. W. Fan, “Analytical method for singularity configuration analysis of a new type hybrid virtual axis machine tool,” Journal of Sichuan University, 2002, Vol. 34,pp.9-12.

[5] H. T. Liu, T. Huang, "Kinematic design of 5-DOF hybrid robot with large workspace/limb-stroke ratio,” Chinese Journal of Mechanical Engineering, 2007, Vol. 43, pp.14-19.

[6] F. Gao, X. J. Liu, "Research on robot mechanism with CAD,” Journal of Mechanical Engineering, 2000, Vol. 2, pp.51-53.

[7] C. M.Gosselin, "On the Kniematic Design of Spherical 3-DOF Parallel Manipulators,” International Journal of Robot Res.,1993, 
Vol.12, pp.394-402.

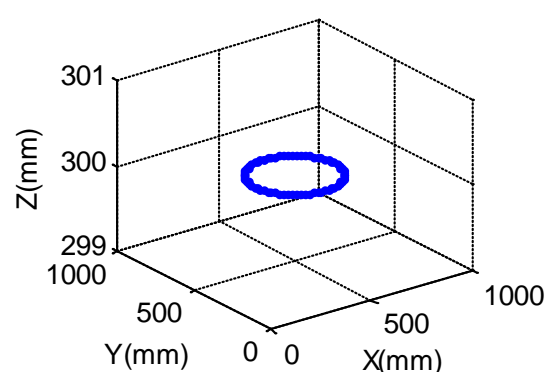

(a) Position of point $\mathrm{P}$

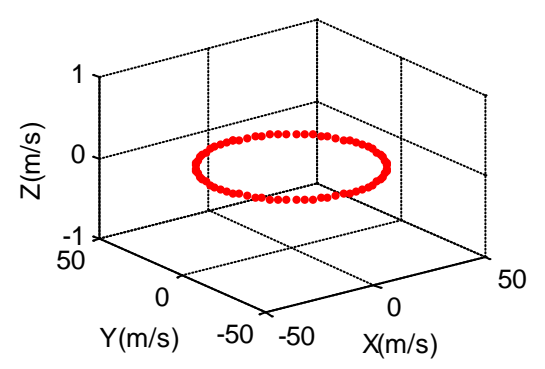

(b) Velocity of point $P$

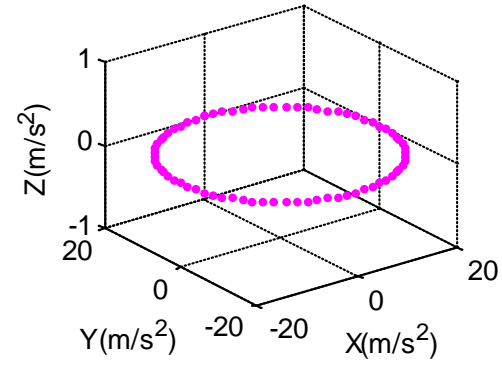

(c) Acceleration of point $\mathrm{P}$

FIGURE II. POSITION AND VELOCITY AND ACCELERATION OF POINT P

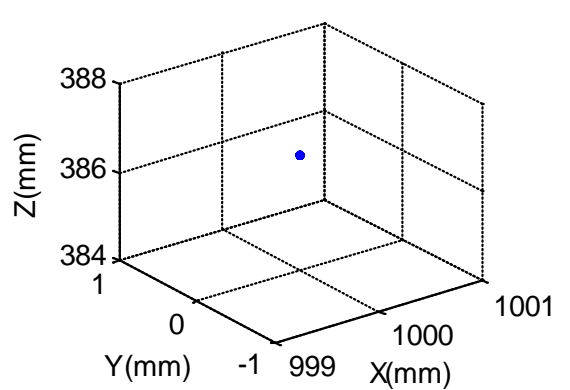

(a) Position of point $C_{1}$

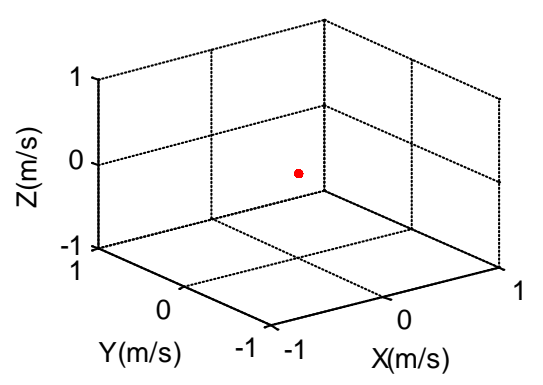

(b) Velocity of point $C_{1}$

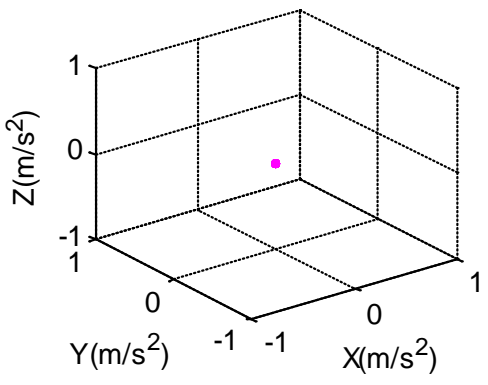

(c) Acceleration of point $C_{1}$

FIGURE III. POSITION AND VELOCITY AND ACCELERATION OF THE INPUT SLIDER $C_{1}$

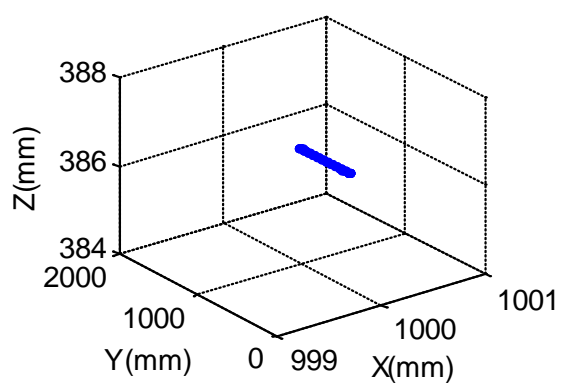

(a) Position of point $C_{2}$

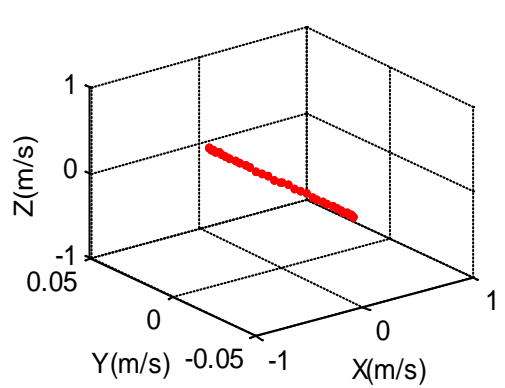

(b) Velocity of point $C_{2}$

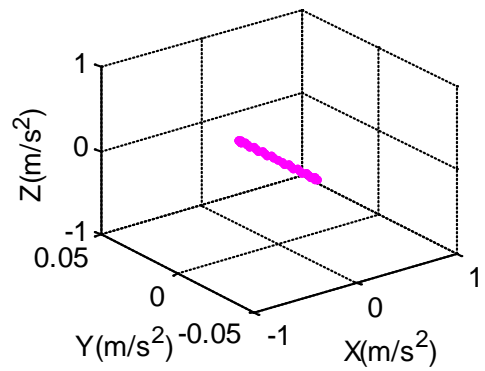

(c) Acceleration of point $C_{2}$

FIGURE IV. POSITION AND VELOCITY AND ACCELERATION OF THE INPUT SLIDER $C_{2}$

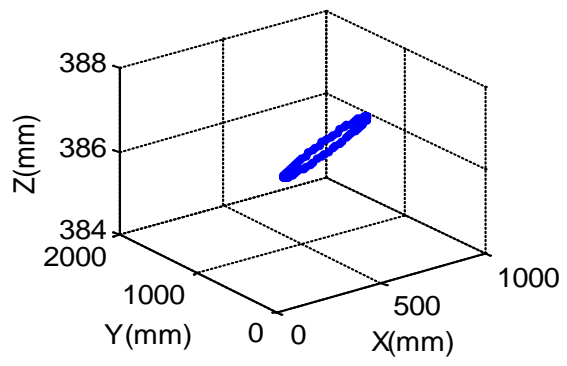

(a) Position of point $C_{3}$

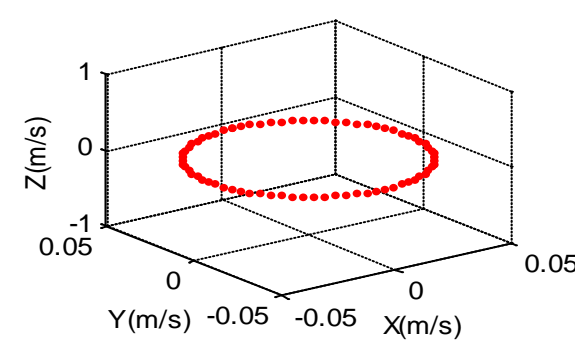

(b) Velocity of point $C_{3}$

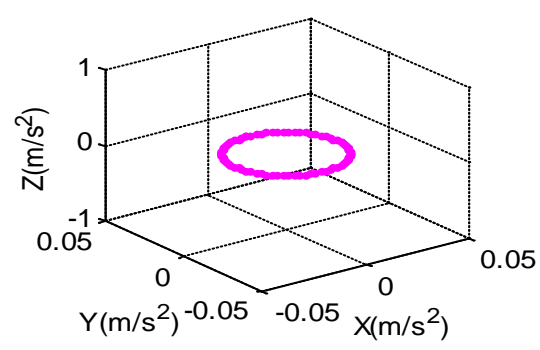

(c) Acceleration of point $C_{3}$

FIGURE V. POSITION AND VELOCITY AND ACCELERATION OF THE INPUT SLIDER $C_{3}$ 


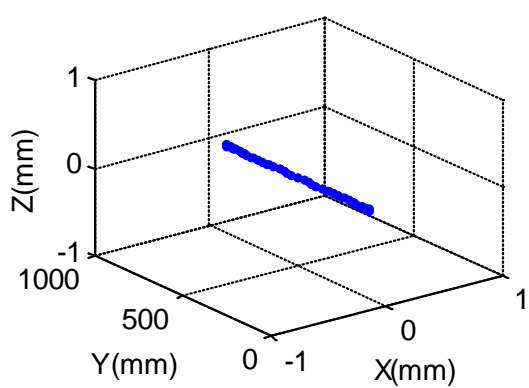

(a) Position of point $C_{4}$

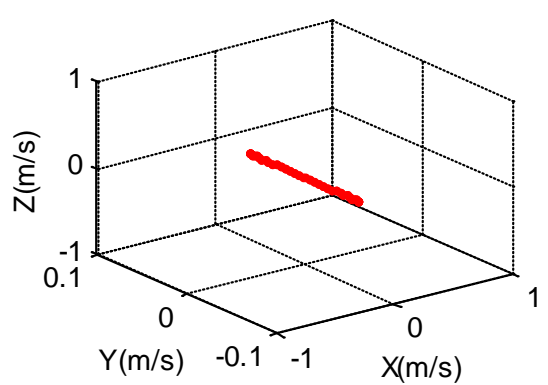

(b) Velocity of point $C_{4}$

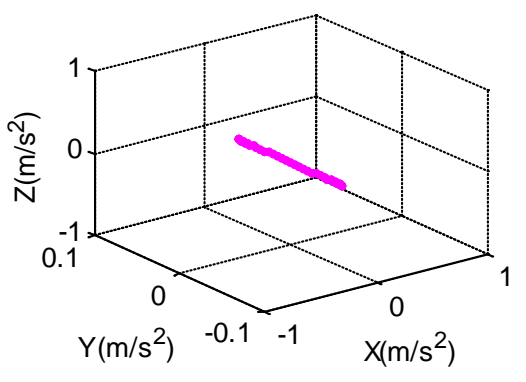

(c) Acceleration of point $C_{4}$

FIGURE VI. POSITION AND VELOCITY AND ACCELERATION OF THE INPUT SLIDER $C_{4}$

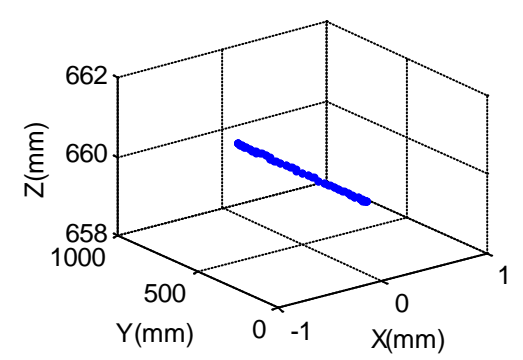

(a) Position of point $C_{5}$

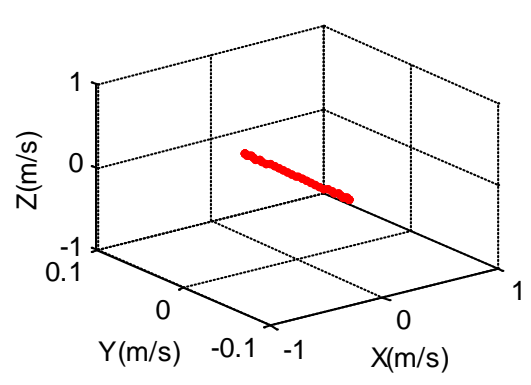

(b) Velocity of point $C_{5}$

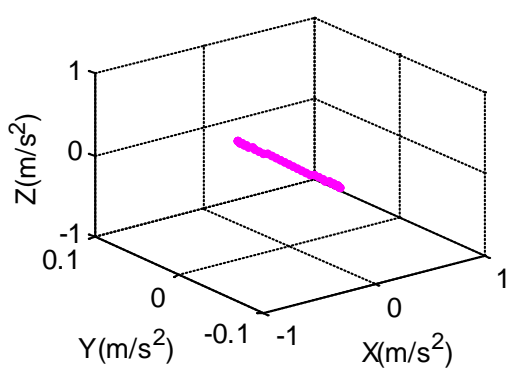

(c) Acceleration of point $C_{5}$

FIGURE VII. POSITION AND VELOCITY AND ACCELERATION OF THE INPUT SLIDER $C_{5}$

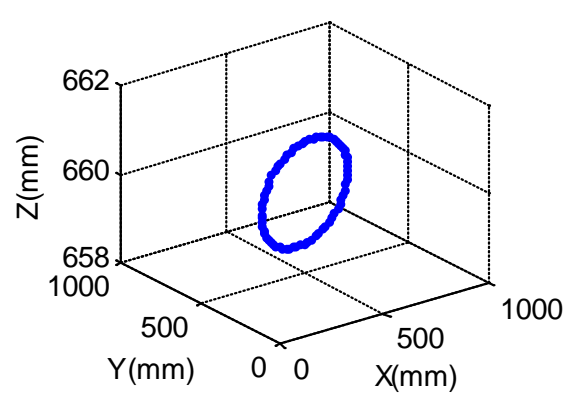

(a) Position of point $C_{6}$

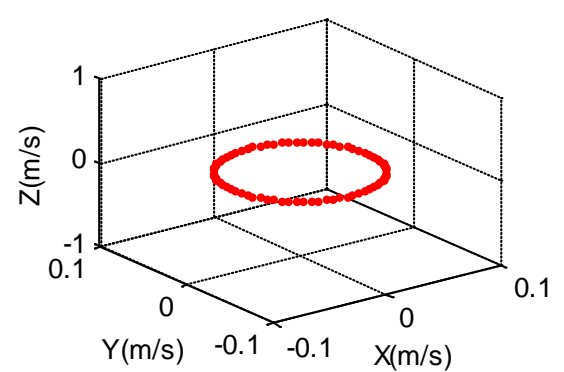

(b) Velocity of point $C_{6}$

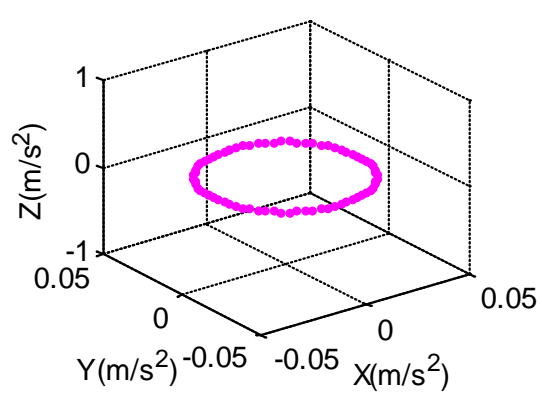

(c) Acceleration of point $C_{6}$

FIGURE VIII. POSITION AND VELOCITY AND ACCELERATION OF THE INPUT SLIDER $C_{6}$ 